\title{
Normas de convivencia
}

\author{
Coexistence rules
}

Jacobo Choy Gómez*

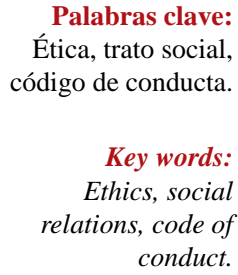

*Adscrito al Servicio de Cirugía General Sanatorio VISA Ciudad de México.

Recibido: 15/04/2016 Aceptado: 27/10/2017

\section{RESUMEN}

Las exigencias sociales e individuales de hoy esperan que todo médico conozca, cumpla y haga cumplir los principios de la ética médica. Es conveniente establecer con coraje y fuerza un conjunto regulatorio de reglas sobre la conducta médica y los derechos de los pacientes. Las normas socialmente reconocidas - como la moda, la tradición, los usos y costumbres- tendrán efecto sobre la conducta en la relación médico-paciente y las normas del trato social, que permitirán establecer confianza en la práctica médica en la institución y en el sector privado. El comportamiento de los miembros de los equipos de salud está relacionado con las actividades de formación de nuevo personal.

\section{ABSTRACT}

Social and individual demands expect every physician to know, comply with and enforce the principles of medical ethics. It is expedient to establish with courage and force a regulatory set of rules on medical conduct and patients' rights. The socially recognized norms — such as fashion, tradition, and customs-will have an effect on the patient-doctor relationship and the norms of social treatment, which will establish trust in medical practice both in the institution and in the private sector. The behavior of the health team members is related to training activities for new staff.

\section{INTRODUCCIÓN}

$\mathrm{L}$ a comunidad le exige al médico suficiente upreparación científica, habilidad en su arte, idoneidad profesional, gran moralidad y cierta proyección social en el ejercicio de sus funciones. La mayor responsabilidad moral de todo médico es conocer, cumplir y hacer cumplir los principios de la ética médica en relación con el paciente y sus familiares. Saber bien estos principios y llevarlos a la práctica cotidiana es la mejor profilaxis para una buena práctica médica. La conveniencia es establecer con valor y fuerza reglamentaria un conjunto de normas sobre conducta médica y derechos del paciente. Estas normas pueden tener fuerza de ley, según la legislación de cada país en relación con el derecho médico. ${ }^{1}$

El paciente se hace cada vez más exigente, y hace valer su derecho a elegir o cambiar libremente su médico y hospital o institución de servicio de salud, sin considerar si forman parte del sector público o privado; esto fue establecido por el Consejo de la Asociación Mundial de los Médicos, ${ }^{2}$ y reafirmado por la 200ª Sesión del Consejo de la AMM, en Oslo,
Noruega, en abril de 2015. El otorgar la libertad de elegir al médico produce un evento en donde participa la familia, amigos, religión y medios de comunicación.

A nivel de la medicina social o institucional, el médico responde solicitando múltiples estudios, con frecuencia innecesarios, para evitar reclamos por omisión o falta de evidencias para su diagnóstico y tratamiento, lo que es una respuesta hacia la medicina defensiva y se contrapone al código de conducta. Este debe guiar sus actividades y acciones con el propósito de contribuir a mejorar la calidad de los servicios y favorecer la satisfacción de los pacientes y la comunidad, así como de los profesionales que los atienden, de manera que se incremente la confianza en las instituciones y su personal.

\section{Norma}

El concepto de norma está en relación con el área en que es aplicada, como anatomía, informática, literatura, matemáticas y meteorología. En este caso, se utilizará el siguiente concepto, que define norma como una regla a la que se 
deben ajustar las conductas, tareas y actividades del ser humano en una determinada sociedad; el conjunto de las mismas compone la parte moral o ética de la cultura.

Es necesario definir la norma jurídica, que es una regla que regula el comportamiento de los individuos en la sociedad y cuyo incumplimiento se encuentra sancionado por el propio ordenamiento contenido en reglamentos; su violación es un acto ilícito. Toda norma jurídica contiene una serie de elementos: ${ }^{3}$

- Supuesto de hecho: actividad humana que da origen a que la norma se cumpla.

- Contenido: mandato o prohibición en que la norma consiste.

- Sanción o efectos de la norma: toda medida desfavorable para la persona a la que se aplique.

- Ordinarias: cumplimiento de la norma.

- Extraordinarias: no se cumple la norma, por lo que existirá una sanción desfavorable para la persona a la que se aplica.

- La pena es un tipo de sanción establecida por el poder positivo del estado.

La norma social es un amplio grupo de normas socialmente reconocidas, como la moda, la tradición, los usos y costumbres, así como actuar de acuerdo a los parámetros de nuestros antepasados para diferenciar lo que está bien de lo que está mal; son impuestas por personas de determinada conducta en la vida social fundada en principios de buena educación, protocolo o cortesía. Su incumplimiento no implica una sanción, aunque sí algún tipo de recriminación o reproche social. En las últimas décadas existe la tendencia a conceptualizar estos comportamientos como «diversos» en vez de como «desviados» de forma adjetiva, tal como el comportamiento informal.

La conducta del médico cuando es servidor público debe apegarse a los principios de legalidad, honradez, lealtad, imparcialidad y eficiencia establecidos en el artículo 113 del título cuarto de la Constitución Política de los Estados Unidos Mexicanos, con el propósito de que impere invariablemente en los servidores públicos una conducta digna que fortalezca a las instituciones públicas y, a su vez, responda a las necesidades de la sociedad. Se publicó en el Diario Oficial de la Federación el 31 de julio de 2002, el Código de Ética de los Servidores Públicos de la Administración Pública Federal. Se estipularon los indicadores de cumplimiento del código de conducta y el método que se seguiría para evaluar anualmente los resultados obtenidos, así como para difundir dichos resultados. Cada dependencia debe contar con su respectivo código de conducta, que tenga un lenguaje claro e incorpore un listado de los valores específicos cuya observancia resulta indispensable.

La conducta del profesional de la salud se encuentra regulada por la Ley Federal de Responsabilidades Administrativas de los Servidores Públicos ${ }^{4}$ en el artículo 8.- Todo servidor público tendrá las siguientes obligaciones, Fracción VI.- Observar buena conducta en su empleo, cargo o comisión, tratando con respeto, diligencia, imparcialidad y rectitud a las personas con las que tenga relación con motivo de éste. Artículo 13.- Las sanciones por falta administrativa consistirán en: I.- Amonestación privada o pública; II.- Suspensión del empleo, cargo o comisión por un periodo no menor de tres días ni mayor a un año; III.- Destitución del puesto; IV.- Sanción económica, e V.- Inhabilitación temporal para desempeñar empleos, cargos o comisiones en el servicio público. Cuando no se cause daños o perjuicios, ni exista beneficio o lucro alguno, se impondrán de seis meses a un año de inhabilitación. Cuando la inhabilitación se imponga como consecuencia de un acto $u$ omisión que implique beneficio o lucro, o cause daños o perjuicios, será de un año hasta diez años si el monto de aquéllos no excede de doscientas veces el salario mínimo general mensual vigente en el Distrito Federal, $y$ de diez a veinte años si excede de dicho límite. Este último plazo de inhabilitación también será aplicable por conductas graves de los servidores públicos. Artículo 14.- Para la imposición de las sanciones administrativas, se tomarán en cuenta los elementos propios del empleo, cargo o comisión que desempeñaba el servidor público cuando incurrió en la falta, que a continuación se refieren. Artículo 48. - Establecer acciones permanentes para delimitar las conductas que en situaciones específicas deberán observar estos en el desempeño de sus empleos, cargos o comisiones. Artículo 49.- La Secretaría, con sujeción a lo previsto en el artículo 48 de la 
ley, emitirá un código de ética que contendrá reglas claras para que, en la actuación de los servidores públicos, impere invariablemente una conducta digna que responda a las necesidades de la sociedad y oriente su desempeño en situaciones específicas que se les presenten, propiciando así una plena vocación de servicio público en beneficio de la colectividad.

\section{Norma de trato social}

Las normas del trato social identifican su decoro ante la sociedad estableciendo sus valores; se relacionan con las normas morales y jurídicas. Existe bilateralidad en la relación médicopaciente. El paciente es un ser social que vive y se desarrolla en sociedad. Las normas del trato social se ponen de manifiesto cuando estamos en sociedad con otra persona. Fuera de esta relación, carecen de sentido. El comportamiento ideal de los profesionales de la salud interpreta las normas morales y de trato social.

En el año 2002, ${ }^{5}$ con el fin de incrementar la confianza en las instituciones y el personal, se formó el Código de Conducta que la Secretaría de Salud, la Subsecretaría de Innovación y Calidad, la Comisión Nacional de Bioética, el Instituto Mexicano del Seguro Social, el Instituto de Seguridad y Servicios Sociales de los Trabajadores al Servicio del Estado, la Coordinación General de los Institutos Nacionales de Salud y la Dirección de Vinculación con Gobierno y Sociedad de la SECODAM ofrecen a todos los profesionales de la salud. En él destaca lo siguiente: la relación profesional será amable, personalizada y respetuosa hacia los pacientes y quien tutele sus derechos, a los que habrá que dirigirse por su nombre, sin diminutivos ni familiaridades; de la misma manera, el equipo de salud se esforzará por establecer vínculos de comunicación efectiva con el paciente y sus allegados, y obtener así su confianza en el personal que los atiende y en su institución. En el artículo 51 de la Ley General de Salud, se establece que los usuarios tendrán derecho a obtener prestaciones de salud oportunas y de calidad idónea y a recibir atención profesional y éticamente responsable, así como trato respetuoso y digno de los profesionales, técnicos y auxiliares. El trato de los integrantes del equipo de salud habrá de ser digno y considerado respecto de la condición sociocultural de los pacientes y sus convicciones personales y morales, con acato a su intimidad y pudor, independientemente de su edad, género y circunstancias personales. El artículo 75 de la Ley General de Salud destaca que el internamiento de personas con padecimientos mentales en establecimientos destinados a tal efecto se ajustará a principios éticos y sociales, además de los requisitos científicos y legales que determine la Secretaría de Salud y establezcan las disposiciones jurídicas aplicables. Es importante proporcionar la información clara, oportuna y veraz que a cada uno corresponda, entregada con mesura y prudencia, considerando la aguda sensibilidad de los pacientes y sus familiares, procurando favorecer su aceptación gradual, sobre todo a las noticias desfavorables. Asimismo, evitar establecer relaciones románticas o sexuales con los pacientes y familiares, explotando la confianza, emociones o influencia derivadas de sus vínculos profesionales. Los profesionales de la salud deberán evitar comentar en público aspectos médicos y sociales de los pacientes o de la institución, resaltando su propia persona. También, habrán de constituirse en ejemplo de probidad profesional ante los pacientes y de acatamiento a la vida sana libre de adicciones y practicar algún tipo de ejercicio físico. La presentación, el arreglo debido y el lenguaje empleado por el personal que presta servicios de salud son considerados importantes en la relación con los pacientes. Se debe, también, preparar a los pacientes que irremediablemente van a morir, así como a sus familiares, junto con los demás miembros del equipo de salud, para que con lucidez mental dispongan de sus bienes, se despidan de sus seres queridos y resuelvan, en su caso, sus problemas de conciencia y asuntos religiosos, respetando su credo. El personal de salud respetará profundamente el secreto profesional confiado al médico y no deberá comentar con ligereza o desdén hechos de la vida de los pacientes, aunque aparentemente no tengan importancia. 6,7

\section{Estándares en la formación}

Se trata del comportamiento de los miembros de los equipos de salud relacionado con las actividades de formación de nuevo personal. La formación del profesional de la salud se hará 
con mesura, prudencia y calidez, respetando la dignidad humana. ${ }^{8}$ Se deberá instruir sobre la dependencia, vulnerabilidad y temor del paciente. No se permitirá, conforme a las normas institucionales y programas académicos, la práctica inexperta o sin supervisión de los alumnos de los diversos grados de los grupos pertenecientes a las diferentes carreras de la salud bajo su responsabilidad. Se inculcará a los alumnos una actitud de servicio y de trato cordial y respetuoso a los pacientes y sus familiares, a los compañeros, a los superiores $y$, en general, al personal que colabora en las actividades asistenciales, cuidando que la formación que reciban sea integral. No se deberán discutir los casos clínicos frente a los pacientes o el personal administrativo, así como evitar comentarios que puedan dañar la sensibilidad de los propios pacientes o dar lugar a interpretaciones indebidas. En relación con lo anterior, el contenido de los artículos 87 de la Ley General de Salud, 24 de la Ley General de Educación, 9. ${ }^{\circ}$; 23, fracción VIII, y el título VII de la Ley Reglamentaria del artículo $5 .^{\circ}$ constitucional y las Bases para la Instrumentación del Servicio Social de las Profesiones para la Salud, establece que los pasantes de medicina que realizan la prestación del servicio social mediante su participación en las unidades aplicativas del primer nivel de atención, y prioritariamente en áreas de menor desarrollo económico y social, deberán ser supervisados por sus tutores académicos en todo momento.

\section{REFERENCIAS}

1. Herrera-Medina NE, Gutiérrez-Malaver ME, Ballesteros-Cabrera M, Izzedin-Bouquet R, GómezSotelo AP, Sánchez-Martínez LM. Representaciones sociales de la relación médico paciente en médicos y pacientes en Bogotá, Colombia. Rev Salud Pública. 2010; 12: 343-355.

2. Nuno-Vieira D, Carracedo A. Las ciencias forenses en el siglo XXI y la necesidad de cooperación global. Cuad Med Forense. 2011; 17: 5-5.

3. Barbero-Gutiérrez J, Sánchez-Caballero M, MartínCortecero JM. Secreto profesional y riesgo vital para un tercero identificado: metodología de análisis ético en torno a un caso. Rev Asoc Esp Neuropsiq. 2013; 33: 555-573.

4. Ley Federal de Responsabilidades Administrativas de los Servidores Públicos. Última reforma publicada DOF 14-07-2014.

5. Comisión Nacional de Bioética. Código de Conducta para el Personal de Salud 2002. Disponible en: http://www.salud.gob.mx/unidades/cdi/documentos/ bioetica/codigo_conducta.html

6. Mariñelarena-Mariñelarena J, Cote-Estrada L. Códigos de ética en medicina. Su transitar por la historia. Cir Gen. 2010; 32: 49-52.

7. Código de Conducta de los Servidores Públicos de la Secretaría de la Defensa Nacional.

8. Gual A. Responsabilidad social en la formación de los médicos. Educ Méd. 2011; 14: 133-135.

Correspondencia:

Jacobo Choy Gómez

E-mail: jacobo.choy@gmail.com 